\title{
Monopoles Loaded with 3-D-Printed Dielectrics for Future Wireless Intra-Chip Communications
}

\author{
Junqiang Wu, Graduate Student Member, IEEE, Avinash Kodi, Senior Member, IEEE, Savas Kaya, \\ Senior Member, IEEE, Ahmed Louri, Fellow, IEEE, and Hao Xin, Senior Member, IEEE
}

\begin{abstract}
We propose a novel antenna design enabled by 3-D printing technology for future wireless intra-chip interconnects aiming at applications of multicore architectures and system-on-chips (SoCs). In our proposed design we use vertical quarter-wavelength monopoles at $160 \mathrm{GHz}$ on a ground plane to avoid low antenna radiation efficiency caused by the silicon substrate. The monopoles are surrounded by a specially-designed dielectric property distribution. This additional degree of freedom in design enabled by 3-D printing technology is used to tailor the electromagnetic wave propagation. As a result, the desired wireless link gain is enhanced and the undesired spatial crosstalk is reduced. Simulation results show that the proposed dielectric loading approach improves the desired link gain by 8-15 $\mathrm{dB}$ and reduces the crosstalk by $9-23 \mathrm{~dB}$ from $155 \mathrm{GHz}$ to $165 \mathrm{GHz}$. As a proof-of-concept, a $60 \mathrm{GHz}$ prototype is designed, fabricated and characterized. Our measurement results match the simulation results and demonstrate $10-18 \mathrm{~dB}$ improvement of the desired link gain and 10-30 dB reduction in the crosstalk from $55 \mathrm{GHz}$ to 61 GHz. The demonstrated transmission loss of the desired link at a distance of $17 \mathrm{~mm}$ is only $15 \mathrm{~dB}$, which is over $10 \mathrm{~dB}$ better than the previously reported work.
\end{abstract}

Index Terms - antennas; electromagnetic propagation; interconnect; intra-chip communication; multiprocessor interconnection; network on chip; 3-D printing

\section{INTRODUCTION}

$\mathrm{T}$ HE continuing reduction in CMOS feature size has resulted in sub-10 nm (Intel just announced a 7-nm fab in Phoenix, Arizona [1]) which will allow integration of kilo-core (1000

Manuscript received April 6, 2017; revised September 11, 2017; accepted September 21, 2017. Date of publication; date of current version. This work was supported in part by funding from National Science Foundation under Grant ECCS-1408271, CCF-1513923, CCF-1054339 (CAREER), CCF-1420718, CCF-1318981, CCF1513606, CCF-1547034, CCF-1547035, CCF-1565273, and CCF-1600820.

J. Wu was with the Department of Electrical and Computer Engineering, University of Arizona, Tucson, AZ 85721 USA and is now with Maxim Integrated Inc., Beaverton, OR 97005, USA (e-mail: jqwu@email.arizona.edu).

A. Kodi and S. Kaya are with the Department of Electrical Engineering and Computer Science, Ohio University, Athens, OH 45701 USA (e-mail: kodi@ohio.edu; kaya@ohio.edu).

A. Louri is with the Department of Electrical and Computer Engineering, George Washington University, Washington, DC 20052 USA (e-mail: louri@gwu.edu)

H. Xin is with the Department of Electrical and Computer Engineering and the Department of Physics, University of Arizona, Tucson, AZ 85721 USA (e-mail:hxin@ece.arizona.edu).

Color versions of one or more of the figures in this paper are available online at http://ieeexplore.ieee.org.

Digital Object Identifier cores) processors in future multicore chips. However, as the width of traditional on-chip RC line interconnects also scales down, the extreme-scaling poses a significant performance challenge for traditional on-chip interconnects: Narrower metal interconnects are plagued with a larger intrinsic R-C time delay and energy losses due to higher resistive and capacitive parasitics [2]-[4]. Moreover, as the core counts keep increasing, traditional wired network-on-chip (NoC) solutions, which used to provide the required connectivity in multicore architectures, face increased latency and power consumption [5]. Therefore, kilo-core on-chip interconnects are a very critical design challenge for scaling these architectures [2]-[4], [6], [7].

Wireless interconnects have been proposed as a promising complementary alternative to the traditional metallic-based RC line interconnects. Wireless links rely on electromagnetic waves propagating at the speed of light, which avoid the performance degradation, especially latency, caused by the traditional RC line interconnect scaling. Further, wireless links can provide cost-effective long-range and high-bandwidth direct links between distant cores, thus circumventing multi-hop latencies [5]. Consequently, Wireless NoCs (WiNoCs) are a promising alternative to cope with both latency and power efficiency concerns of future multicore architectures [8]-[10].

The design of on-chip antennas is one of the biggest challenges for wireless interconnects [11] due to the following two reasons. First, the CMOS substrate, i.e., the silicon substrate, has a low resistivity (thus high loss) and a high dielectric constant [12]. When frequency reaches tens of $\mathrm{GHz}$, silicon substrate attracts and confines most of the electromagnetic energy, making the antenna radiation efficiency thus gain very low [13], [14]. For example, in [15], $15 \mathrm{GHz}$ on-chip transmitters, receivers and zig-zag antennas have been demonstrated. The intra-chip communication performance, however, is limited, mainly because of the inadequate gain of the antennas. At $15 \mathrm{GHz}$, the antenna pair suffer a high transmission loss of $45 \mathrm{~dB}$ for 3.2-mm separation [15]. Second, even without the impact from the silicon substrate, large path losses and undesired crosstalk between antennas may significantly limit the $\mathrm{WiNoC}$ performance.

To reduce the impact from the silicon substrate on on-chip antennas, a number of technologies have been proposed. In [16], a CMOS on-chip slot antenna at $140 \mathrm{GHz}$ backed with an extremely thin cavity formed by two CMOS inner metal layers and vias in between is proposed. The cavity protects the 
electromagnetic energy from being absorbed by the substrate thus enhances the radiation efficiency of the slot antenna. However, the bandwidth of the antenna is only $5 \mathrm{GHz}$, which is mainly caused by the cavity's flatness. In [17], a high dielectric constant $\left(\varepsilon_{\mathrm{r}}=38\right)$ rectangular dielectric resonator excited by an $\mathrm{H}$-slot antenna is achieved based on the IBM SiGe5AM process. The gain of this antenna is $1 \mathrm{dBi}$ at $35 \mathrm{GHz}$. However, the radiation is mainly towards the vertical direction, making it less attractive for intra-chip communication that requires horizontal communication.

In this work, we propose to use vertical quarter-wavelength monopoles on a ground plane at $160 \mathrm{GHz}$ for wireless intra-chip interconnects. The ground plane separates the silicon substrate from the antenna, eliminating the substrate effects and enhancing radiation efficiency. More importantly, well-designed artificial dielectrics surrounding the monopoles enabled by 3-D printing technology are employed to enhance the performance of desired wireless links and reduce undesired cross-talk. The resulting antenna design leads to a more power-efficient and less interference-prone wireless interconnects. We conduct both a full-wave 3-D electromagnetic simulation and a proof-of-concept experimental design study to validate the proposed approach. To the best of our knowledge, this is the first work that proposes the use of the 3-D printing technology to design high-performance antennas for wireless interconnects aiming at multicore architectures applications.

This paper is organized as follows. Section II presents the NoC architecture, which utilizes wireless interconnect for long range intra-chip interconnects. Section III explores the dielectric loading method for the vertical monopoles at 160 $\mathrm{GHz}$ as intra-chip wireless interconnects; next, the design of a prototype at $60 \mathrm{GHz}$ for the experimental validation is presented. Section IV presents the fabrication and measurement results, which demonstrate good agreement with the simulation results. Section V provides a detailed discussion centered on the latency comparison of the proposed wireless interconnects and traditional RC line interconnects. Finally, Section VI concludes the paper.

\section{NETWORK-ON-CHIP ARCHITECTURE}

The antenna structures proposed are for a novel Optical-Wireless NoC (OWN) architecture for kilo-core computing that utilizes both optical and wireless interconnects [18]. Wireless interconnects reduce the hop count between the optically connected clusters, leading to improved performance and more efficient utilization of the finite wireless bandwidth. Our previous results indicate that OWN architecture consumes $30.36 \%$ less energy, and improves throughput by $8 \%$ over wireless-alone architectures and obtains $35.5 \%$ less area than optical-alone architectures [18].

The OWN architecture is a tile-based architecture with each tile consisting of four processing cores and their private L1 instruction and data caches, a shared L2 cache and a network interface or router. Each tile is located within a cluster, which consists of 16 such tiles (64 cores), as shown in Fig. 1. Four clusters form the 256-core OWN architecture, which has an area of about $20 \times 20 \mathrm{~mm}^{2}$ (note that the designs shown in this work can be scaled to other chip sizes such as $50 \times 50 \mathrm{~mm}^{2}$ ). Intra-cluster communication is implemented using optical interconnects whereas inter-cluster communication is facilitated by 16 wireless links operating within $20 \mathrm{GHz}$ bands, between 60 and $380 \mathrm{GHz}$.

To facilitate inter-cluster communication, we propose to use wireless routers N1-N8 and C1-C4 as illustrated in Figure 1. Wireless router N1 (top-left cluster) communicates with N2 (top-right cluster); wireless router N3 (top-left cluster) communicates with wireless router $\mathrm{N} 4$ (bottom-left cluster) and so on. Therefore, wireless routers N1-N8 enable neighboring inter-cluster communication (same color). Wireless routers $\mathrm{C} 1$ (top-left cluster) communicates diagonally with C3 (bottom-right cluster) and $\mathrm{C} 2$ communicates with $\mathrm{C} 4$. That is to say, corner-to-corner $(\mathrm{C} 2 \mathrm{C})$ wireless links (i.e. C1-to-C3 and $\mathrm{C} 2$-to-C4) are desired, whereas edge-to-edge (E2E) wireless links (i.e. C1-to-C2, C2-to-C3, C3-to-C4, and C4-to-C1) are deemed undesired crosstalk/inference. This work focuses on providing antenna solutions for wireless router $\mathrm{C} 1-\mathrm{C} 4$, which are at the vertices of a $14 \times 14 \mathrm{~mm}^{2}$ square. The antennas work at $160 \mathrm{GHz}$, which act as one frequency channel for the OWN architecture. Under the assumption of Quadrature Phase Shift Keying (QPSK) modulation that allows up to $2 \mathrm{bits} / \mathrm{s} / \mathrm{Hz}$ spectral efficiency, $8 \mathrm{GHz}$ frequency bandwidth is expected for the antennas so that $16 \mathrm{Gbps}$ throughput is possible for the channel.

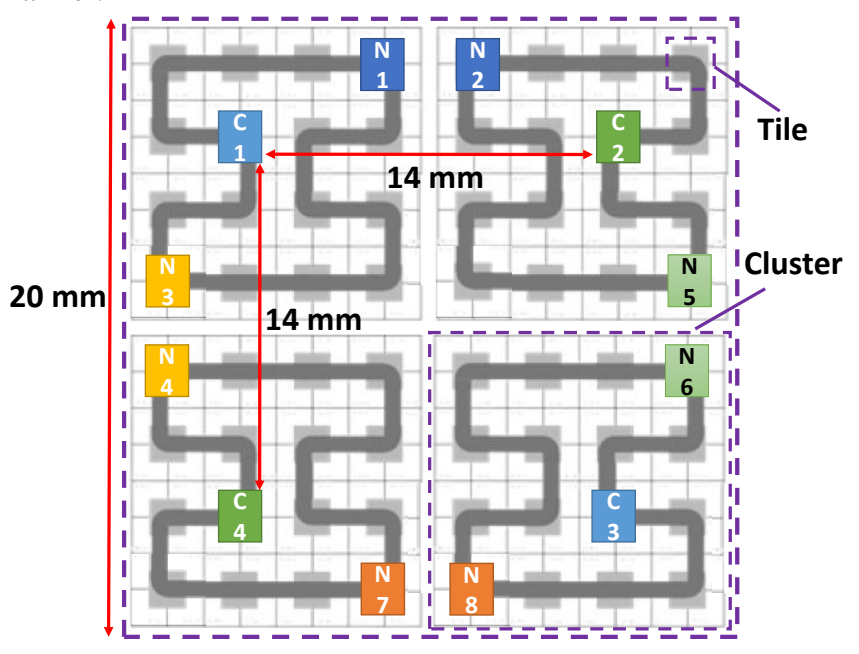

Fig. 1. 256-Core OWN architecture. Routers with the same color communicate with each other.

\section{Design Methodology}

\section{A. $160 \mathrm{GHz}$ Antenna Design for Intra-Chip Interconnect}

In this section we preset the design of the vertical quarter-wavelength antennas operating at $160 \mathrm{GHz}$. In this case, the ground plane helps eliminate the impact from the lossy silicon substrate. Such advantage is verified using a HFSS simulation with the model shown in Fig. 2(a). A monopole antenna (PEC) with a $\lambda_{0} / 4$ length and a $0.1 \mathrm{~mm}$ diameter stands at the center of a $6 \times 6 \mathrm{~mm}^{2}$ ground plane (PEC). Beneath the ground plane is a $290 \mu \mathrm{m}$ thick silicon substrate $\left(\varepsilon_{\mathrm{r}}=11.9, \mu_{\mathrm{r}}=1\right.$, and $\rho=10 \Omega \cdot \mathrm{cm}$; standard 0.18 -um CMOS process substrate thickness). The simulation shows that the antenna has a gain of 
$5.1 \mathrm{dBi}$ at $160 \mathrm{GHz}$, as illustrated in Fig. 2(b), which is close to the theoretical gain $(5.19 \mathrm{dBi})$ of a quarter-wavelength monopole. The simulated radiation efficiency is $99.2 \%$, showing very little loss caused by the silicon substrate. Compared with previously reported on-chip zig-zag dipoles that have -8 to $-10.5 \mathrm{dBi}$ gain [13], the monopole antenna is free from the impact of the substrate effect and achieves much higher gain.

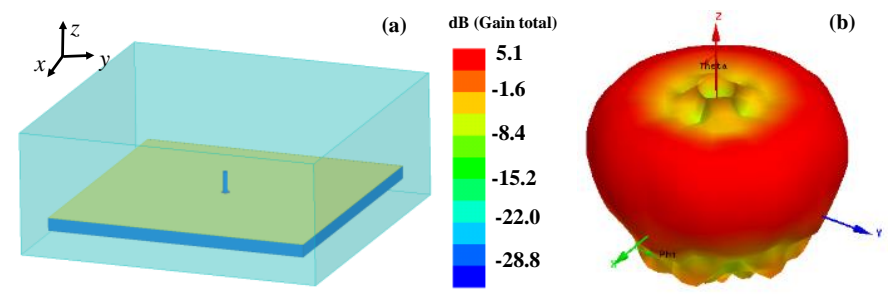

Fig. 2. (a) The HFSS model of a quarter-wavelength monopole antenna on a finite ground $\left(6 \times 6 \mathrm{~mm}^{2}\right)$ in the air box (radiation boundaries). The blue part is the $290 \mu \mathrm{m}$ thick silicon substrate. (b) The radiation pattern of the monopole antenna at $160 \mathrm{GHz}$.
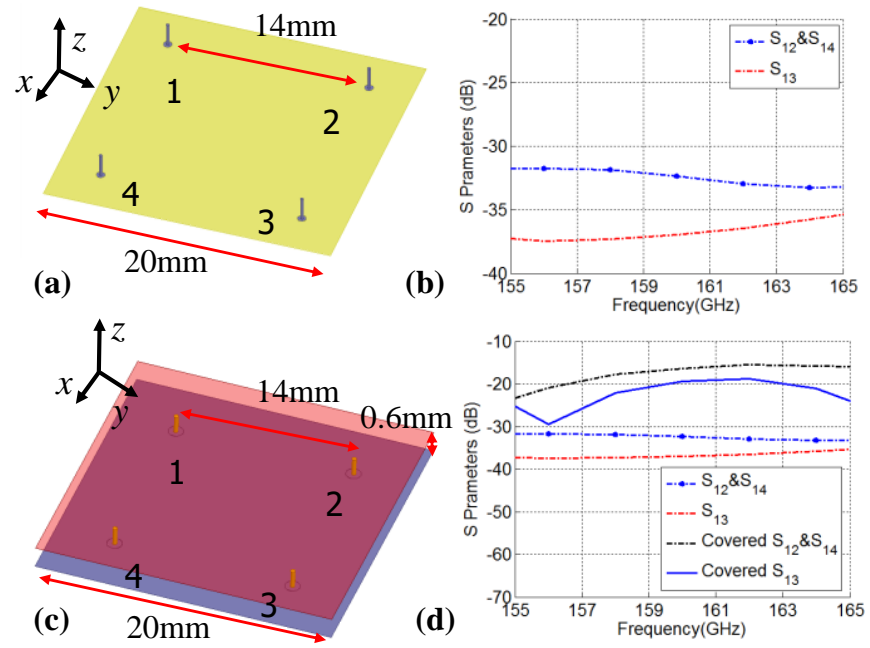

Fig. 3. (a) The HFSS model of four quarter-wavelength monopole antennas on a finite ground. (b) The $S$-parameters of the monopole antennas in Fig. 3(a). (c) The HFSS model of four quarter-wavelength monopole antennas with a metallic cover $0.6 \mathrm{~mm}$ above the ground plane. The metallic cover could be included in the package of the silicon die. (d) The $S$-parameters of monopole antennas with and without the metallic cover.

As illustrated in Fig. 1, the wireless routers are at the vertices of a $14 \times 14 \mathrm{~mm}^{2}$ square. An HFSS model corresponding to the wireless $\mathrm{NoC}$ is setup as illustrated in Fig. 2(a). The four monopoles are located at the vertices of a $14 \times 14 \mathrm{~mm}^{2}$ square. Each monopole is $\lambda_{0} / 4(0.47 \mathrm{~mm})$ long at $160 \mathrm{GHz}$ with a diameter of $0.1 \mathrm{~mm}$. The ground size is $20 \times 20 \mathrm{~mm}^{2}$. The silicon substrate has little impact on the antenna performance, as shown in the single monopole example in Fig. 3(a). Therefore, the substrate is not included in the model for the sake of computational efficiency. The simulation results are shown in Fig. 3(b). It is observed that $S_{13}$ is smaller than $S_{12}$, which is due to the larger distance between Antenna 1 and Antenna 3. Next, a $20 \times 20 \mathrm{~mm}^{2}$ metallic cover is placed $0.6 \mathrm{~mm}$ above the ground plane to help confine radiation in the horizontal plane, as shown in Fig. 3(c). It can be observed in Fig. 3(d) that about $10 \mathrm{~dB}$ improvement is achieved for $\mathrm{C} 2 \mathrm{C}$ and $\mathrm{E} 2 \mathrm{E}$ wireless link gain.
To enhance the desired $\mathrm{C} 2 \mathrm{C}$ link gain and suppress the undesired E2E crosstalk, dielectric loading technique based on 3-D printing technology can be utilized [19]. The space surrounding the monopoles (between the metallic cover and ground) is digitized into ideal dielectric blocks, i.e. unit cells with distinct dielectric constant, $\varepsilon_{\mathrm{r}}$, as illustrated in Fig. 4(a). The inhomogeneity around the monopoles will be utilized to control the propagation of EM wave between the metallic cover and ground, which in turn enhance the desired links and suppresses

crosstalk.

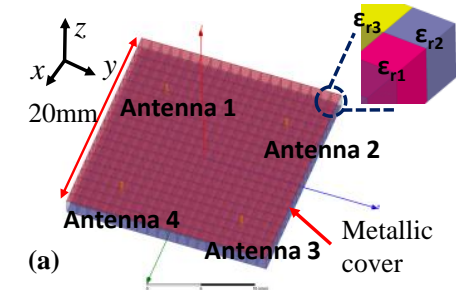

(b)
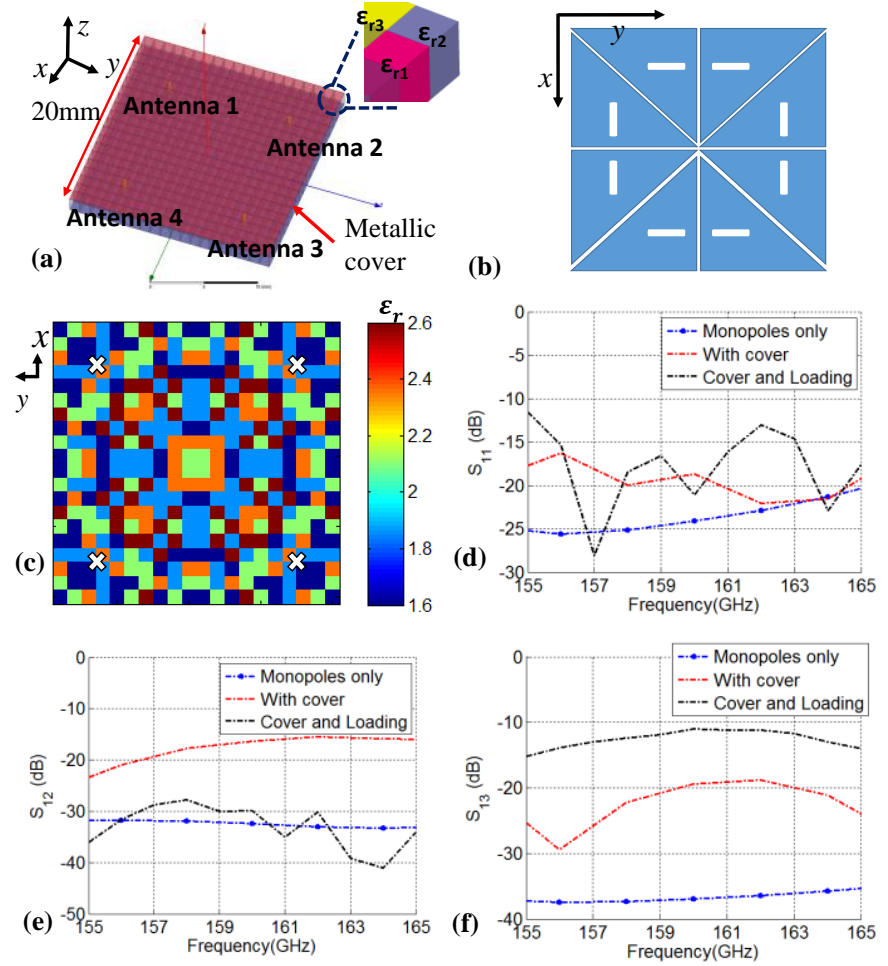

Fig. 4. (a) The HFSS model of four quarter-wavelength monopoles on finite ground with both a metallic cover and unit cells (ideal dielectric blocks) with assigned distinct $\varepsilon_{\mathrm{r}}$. (b) The symmetry used in the $\varepsilon_{\mathrm{r}}$ distribution to reduce optimization parameters by a factor of eight; The symmetry is with respect to the $x$-axis, $y$-axis, and the diagonals. (c) The $\varepsilon_{\mathrm{r}}$ distribution surrounding the 160 $\mathrm{GHz}$ monopoles. The white crosses indicate the location of the monopoles. The $S$ parameters comparison of monopoles only, monopoles with metallic cover, and monopoles with both metallic cover and dielectric loading. (d) $S_{11}$; (e) $S_{12}$; (f) $S_{13}$.

For the $160 \mathrm{GHz}$ antennas, each monopole is $0.36 \mathrm{~mm}$ long (slightly less than $1 / 4 \lambda_{0}$ ) so that the resonance is in the 155-165 $\mathrm{GHz}$ range with the loaded dielectric. The dielectric loading space is discretized into $20 \times 20$ identical blocks, whose dimensions are $1 \times 1 \times 0.6 \mathrm{~mm}^{3}$. The $\varepsilon_{\mathrm{r}}$ of each block can be any value from 1.6 to 2.6 with a 0.25 increment. By optimizing the $\varepsilon_{\mathrm{r}}$ spatial distribution with genetic algorithm (GA), the goal of enhancing $\mathrm{C} 2 \mathrm{C}$ antenna communication and reducing $\mathrm{E} 2 \mathrm{E}$ antenna communication can be achieved in the targeting frequency range of $155-165 \mathrm{GHz}$. It should be noted that the relatively small $\varepsilon_{\mathrm{r}}$ range is chosen to restrict the optimization parameter space thus simplify the optimization problem.

The MATLAB based GA code is utilized to control ANSYS HFSS for optimization of the $\varepsilon_{\mathrm{r}}$ distribution. To reduce the optimization parameters by a factor of eight, a symmetry of the $\varepsilon_{\mathrm{r}}$ distribution with respect to the $x$-axis, $y$-axis, and the diagonals as illustrated in Fig. 4(b) is applied. It should be noted that the E2E link gain is $S_{12}=S_{14}=S_{23}=S_{34}$ and the C2C 
link gain is $S_{13}=S_{24}$ under this symmetry. The GA optimization is defined as follows:

- Objective function: fitness value as large as possible (stop the optimization process when there is no significant improvement, e.g., $\Delta$ fitness $<0.3$ after tens of generations)

- Fitness function: fitness $=\left[S_{13}(f)-S_{12}(f)\right]$, where $f \in[155$ $\mathrm{GHz}, 165 \mathrm{GHz}]$

- Constraints: a $\varepsilon_{\mathrm{r}}$ range of 1.6-2.6 with a discretization of 0.1

- Crossover probability and method: 100\%; one-point crossover

- Mutation probability: 0.1

- Mutation type: generating a random $\varepsilon_{\mathrm{r}}$
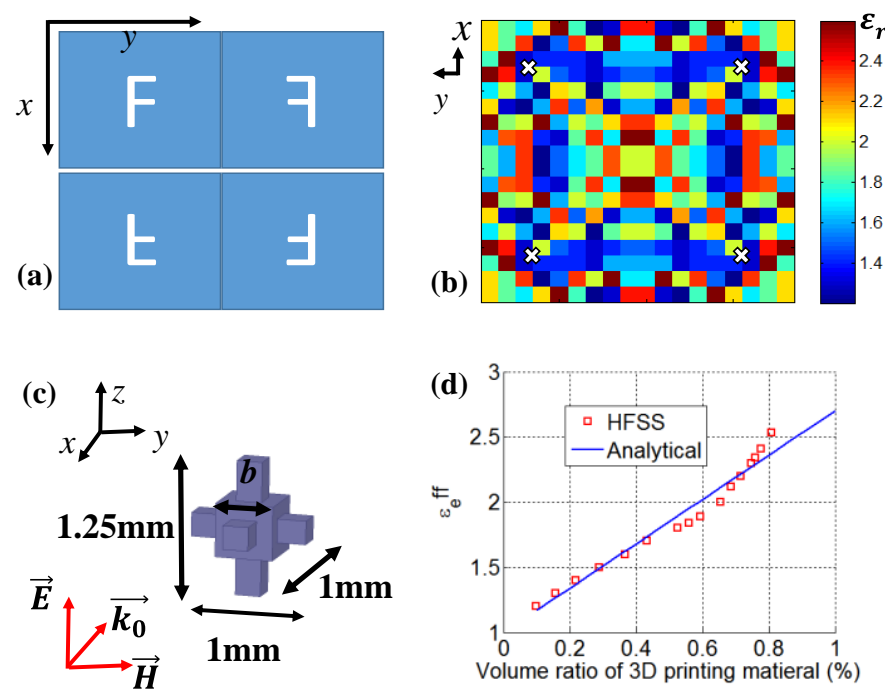

Fig. 5. (a) The symmetry used in the $\varepsilon_{\mathrm{r}}$ distribution to reduce optimization parameters by a factor of four. The symmetry is with respect to the $x$ and $y$-axes. (b) The $\varepsilon_{\mathrm{r}}$ distribution surrounding the $60 \mathrm{GHz}$ monopoles. The white crosses show the location of the monopoles. (c) The unit cell structure of the designed dielectric distribution. (d) The effective dielectric constant of unit cells with different polymer cube size based on analytical calculation and HFSS simulation.

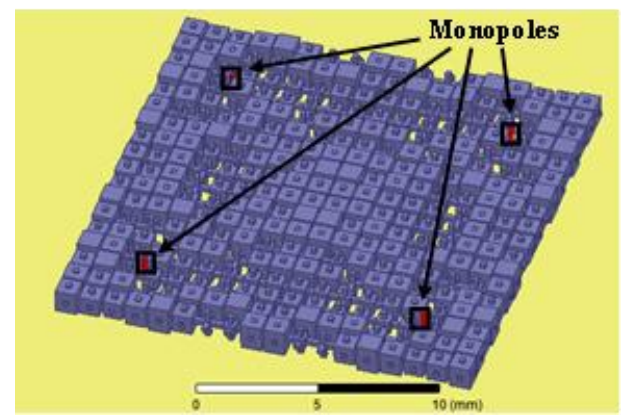

Fig. 6. The HFSS model of the $60 \mathrm{GHz}$ antenna prototype showing the monopoles and 3-D-printed polymer structure.

A population size of 3 is used in the GA for efficient and parallel simulations. Although GA usually works well when the population size is large, we are able to achieve the desired results because the range of $\varepsilon_{\mathrm{r}}$ is not large. The optimized $\varepsilon_{\mathrm{r}}$ distribution is illustrated in Fig. 4(c). The optimization results of S parameters are shown in Fig. 4(d)-(f). It is obvious that the optimized inhomogeneous dielectric distribution improves $S_{13}$ (desired link) by 8-15 $\mathrm{dB}$ compared to the antennas with only metallic cover. Moreover, it suppresses $\mathrm{S}_{12}$ (undesired crosstalk) by $9-23 \mathrm{~dB}$. Consequently, the loaded dielectrics help boost the wireless link efficiency and reduce interference.

\section{B. $60 \mathrm{GHz}$ Antenna Prototype Design}

As a proof-of-concept, an antenna prototype working at 60 $\mathrm{GHz}$ is designed and fabricated. The $20 \times 20 \mathrm{~mm}^{2}$ metallic cover is $1.25 \mathrm{~mm}$ above the ground. Four monopoles are located at the vertices of a $12 \times 12 \mathrm{~mm}^{2}$ square. The monopoles are $1 \mathrm{~mm}$ long, which is slightly less than $\lambda_{0} / 4$ at $60 \mathrm{GHz}$. The diameter of the monopole is $0.23 \mathrm{~mm}$. The monopoles are surrounded by 18 $\times 18$ ideal dielectric blocks (i.e., unit cells) that have a size of $1 \times 1 \times 1.25 \mathrm{~mm}^{3}$, corresponding to $0.2 \lambda_{0}$ at $15 \mathrm{GHz}$, which is a good compromise of effective medium approximation and printing accuracy. The $\varepsilon_{\mathrm{r}}$ of the unit cell range is 1.2-2.6 with a 0.1 discretization, which can be conveniently implemented using a 3-D printing technique reported previously [20].

GA is used to optimize the dielectric constant distribution surrounding the monopoles. A symmetry of the $\varepsilon_{\mathrm{r}}$ distribution with respect to $x$ and $y$ axes as illustrated in Fig. 5(a) is used in the simulation such that $S_{13}=S_{24}, S_{12}=S_{34}$, and $S_{14}=S_{23}$, which reduces the optimization parameters by a factor of four. The GA optimization is defined similarly to the $160 \mathrm{GHz}$ optimization except for the following:

- Fitness function: fitness $=\left[S_{13}(f)-\max \left(S_{12}(f), S_{14}(f)\right)\right]$, where $f \in[55 \mathrm{GHz}, 61 \mathrm{GHz}]$

- Constraints: a $\varepsilon_{\mathrm{r}}$ range of 1.1-2.6 with a discretization of 0.1

The optimized $\varepsilon_{\mathrm{r}}$ distribution is illustrated in Fig. 5(b).

Once the spatial $\varepsilon_{\mathrm{r}}$ distribution surrounding the monopoles is achieved, they are realized by 3-D-printed unit cell structures based on the effective medium approximation. The unit cell structure is shown in Fig. 5(c). It consists of the 3-D-printed polymer (dark blue part; $\varepsilon_{\mathrm{r}}=2.7$ and $\tan \delta=0.02$ [20]) and air voids $\left(\varepsilon_{\mathrm{r}}=1\right)$. The 3-D-printed polymer structure includes 6 pillars and a variable polymer cube with edge size $b$. The pillars in the $x$ and $y$ directions are to mechanically connect each neighboring unit cells. The $z$ direction pillar is to offer mechanical support for the top metallic cover. The cross section of the pillars is $0.22 \times 0.22 \mathrm{~mm}^{2}$ so that they are small enough to avoid impacting much the effective dielectric property control while providing adequate mechanical strength for the structure. By changing $b$, or the filling ratio of the polymer material in the unit cell (i.e., mixing polymer and air at different ratios), effective $\varepsilon_{\mathrm{r}}$ from 1 to 2.7 can be obtained. The approximated permittivity of a unit cell is calculated by the analytical effective medium approximation based on the polymer filling ratio $f$ using $\varepsilon_{r}=\varepsilon_{p} \cdot f+1 \cdot(1-f)$, where $\varepsilon_{r}$ is the effective relative permittivity of the unit cell and $\varepsilon_{p}$ is the relative permittivity of the polymer material [20]. Next, based on the Nicholson-Ross-Weir method [21], [22], the calculated effective permittivity is verified by finite-element simulations using HFSS software. Fig. 5(d) plots the effective relative permittivity extracted from the simulated $S$-parameters and that calculated from the filling ratio equation, with respect to the filling ratio $f$. It should be noted that the unit cell simulation assumes an incident plane wave with the $E$ field in the $z$ direction propagating in the $-x$ direction, as illustrated in Fig. 
5(c). Due to the symmetry of the unit cell structure in the $x$ and $y$ directions, the unit cell's effective dielectric constant $\left(\varepsilon_{\text {eff }}\right)$ should be isotropic. Once the unit cell designs are completed, the entire 3-D-printable antenna system is modeled in HFSS as illustrated in Fig. 6.

The optimized results are shown in Fig. 7(a)-(f), in which three cases are compared: the no loading case where there is no dielectric loading around antennas, e.g., Fig. 3(c); the ideal dielectric blocks loading case where the antennas are surrounded by the unit cells assigned by the desired dielectric constant, i.e., Fig. 4(a); the 3-D-printed polymer loading case where the antennas are surrounded by the entire printed dielectric structure including dielectric polymer and pillars, i.e. Fig. 6. Fig. 7(a) shows that the reflection coefficients are below $-10 \mathrm{~dB}$ from $55 \mathrm{GHz}$ to $61 \mathrm{GHz}$ for all three cases. The antenna's $-10 \mathrm{~dB}$ impedance bandwidth becomes slightly larger after dielectric loading from $45 \mathrm{GHz}$ to $65 \mathrm{GHz}$. It is observed in Fig. 7(b) that the loaded dielectric boosts $S_{13}$ by $10-18 \mathrm{~dB}$ from $55 \mathrm{GHz}$ to $61 \mathrm{GHz}$. The undesired cross talk, $S_{21}$ and $S_{14}$, are attenuated by 10-30 dB as shown in Fig. 7(c) and (d). Fig. 7 (e) shows that the figure of merit $(\mathrm{FoM})=\left(S_{13}-S_{12}\right)$ is $10-20 \mathrm{~dB}$ from $55 \mathrm{GHz}$ to $61 \mathrm{GHz}$, in comparison with -10 to $-3 \mathrm{~dB}$ of the no loading case as shown in Fig. 7(f). Good agreement between the ideal dielectric blocks model and the 3-D-printed polymer model can also be observed. Moreover, the dielectric loss caused by the loaded dielectrics is also investigated in the simulation. As shown in Fig. 8, the dielectric loss leads to about $1 \mathrm{~dB}$ degradation of the $\mathrm{C} 2 \mathrm{C}$ link gain $\mathrm{S}_{13}$, which is acceptable.
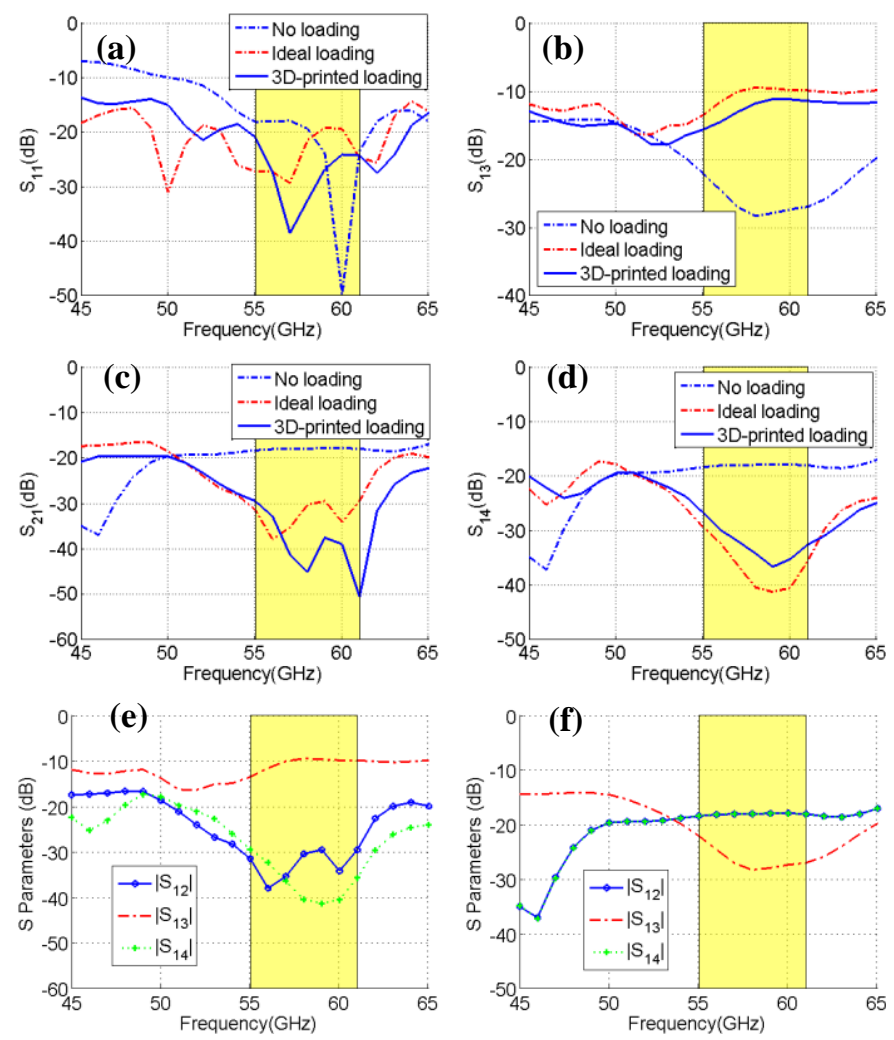

Fig. 7. The $S$-parameters comparison of the no loading, the ideal dielectric blocks loading, and the 3-D-printed polymer loading cases. (a) $S_{11}$; (b) $S_{13}$; (c) $S_{21}$; (d) $S_{14}$. (e) The $S$-parameters of the monopoles loaded with ideal dielectric blocks. (f) The $S$-parameters of the no loading case.

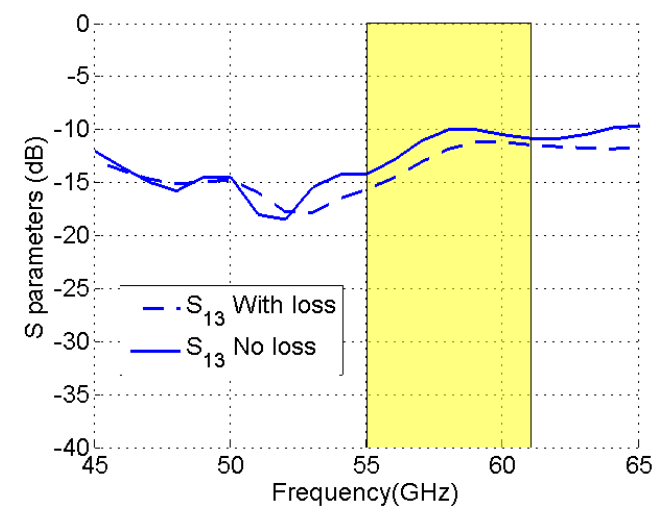

Fig. 8. The $\mathrm{S}_{13}$ comparison of the dielectric loading with and without dielectric loss in the model $(\tan \delta=0.02$ versus $\tan \delta=0)$.

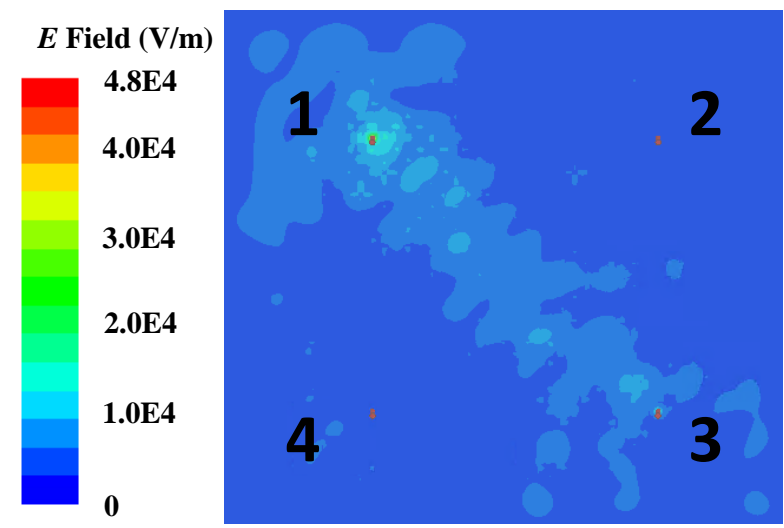

Fig. 9. The simulated $E$ field (complex magnitude) distribution $0.5 \mathrm{~mm}$ above the ground plane when antenna 1 is excited at $60 \mathrm{GHz}$. The red dots are the locations of antennas. The figure shows that directional propagation is achieved between antenna 1 and antenna 3 .

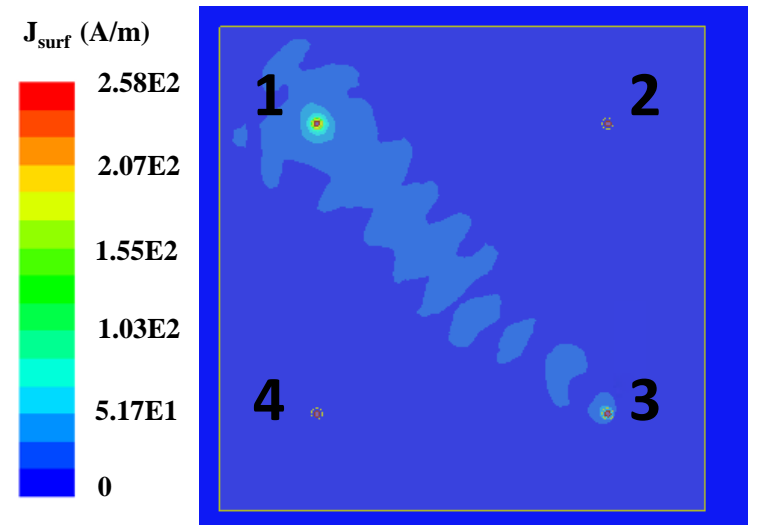

Fig. 10. The simulated surface current distribution (complex magnitude) on the ground plane. The yellow, half-transparent square shows the location of the metallic cover. The red dots are the locations of antennas. The figure shows that directional propagation is achieved between antenna 1 and antenna 3 .

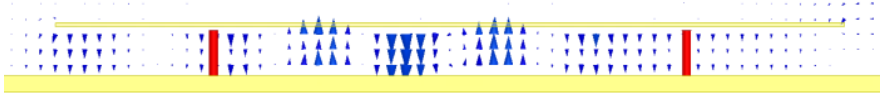

Fig. 11. The simulated vector $E$ field between the metallic cover and ground on the $x \mathrm{oz}$ plane at $60 \mathrm{GHz}$ (the dielectric structure is hidden to better illustrate the field distribution).

The simulated $E$ field distribution (complex magnitude) in the $\mathrm{x}-\mathrm{y}$ plane $0.5 \mathrm{~mm}$ above the ground plane at $60 \mathrm{GHz}$ is shown in Fig. 9. When antenna 1 is excited, electromagnetic 
power travels towards antenna 3 thus the $\mathrm{C} 2 \mathrm{C}$ link gain is improved and E2E link gain is reduced. Fig. 10 shows the surface current distribution (complex magnitude) on the ground plane at $60 \mathrm{GHz}$. It can be observed that the current also concentrates on the diagonal path. If we consider the structure as a parallel plate waveguide, the lowest cutoff frequency for TM or TE modes is $120 \mathrm{GHz}$ and thereby only TEM wave can propagate at $60 \mathrm{GHz}$. To verify this, the vector $E$ field in the $x \mathrm{oz}$ plane at $60 \mathrm{GHz}$ is plotted in Fig. 11. It can be observed that the electric field is mostly in the $\mathrm{z}$ direction.

\section{FABRICATION AND MEASUREMENT}

\section{A. Fabrication}

The conductor part of the antenna prototype (monopoles) is fabricated by precision machining. The monopoles are coaxially fed by $1.85 \mathrm{~mm}(\mathrm{~V})$ connectors through holes in the ground plane. Four holes $(\Phi=0.51 \mathrm{~mm})$ are drilled at the center zone of a piece of copper sheet $\left(152.4 \times 152.4 \mathrm{~mm}^{2} ; 0.4 \mathrm{~mm}\right.$ thick). Four V-connectors accepting $0.23 \mathrm{~mm}$ diameter pins are utilized for antenna feeding. Pins with a diameter $\Phi=0.23 \mathrm{~mm}$ are inserted into the connectors' sockets and carefully soldered to fixate the pins into the sockets, as shown in Fig. 12(a). Next, the V-connectors are attached to the ground via silver epoxy and non-conductive adhesive for electrical and mechanical connection, during which metallic tubes $\left(\Phi_{\text {in }}=0.254 \mathrm{~mm}\right.$ and $\Phi_{\text {out }}=0.45 \mathrm{~mm}$ ) are set in between the pins and holes to help put the pins at the centers of the holes, as illustrated in Fig. 12(b). Afterward, the tubes are removed and pin trimming is performed to obtain $1 \mathrm{~mm}$ long monopoles. The finally fabricated monopoles are illustrated in Fig. 12(c).

Additive manufacturing (AM), or 3-D printing technique, is utilized to fabricate the dielectric blocks surrounding the monopoles. AM enables low cost and rapid prototyping of arbitrary 3-D structures. Multiple applications in electromagnetics have been reported involving different types of AM methods [23]. In this work, the 3-D polymer jetting technique is employed to implement the proposed design. Previously demonstrated electromagnetic components include electromagnetic bandgap structures [24], Terahertz (THz) electromagnetic crystal horn antennas [25], $\mathrm{THz}$ waveguides [26], holograms [27], Luneburg lens antennas [20] and dielectric reflectarray antennas [28].

A commercial polymer jetting 3-D printer, Objet Eden 350, is utilized to fabricate the dielectric part. The resolution is as fine as $84 \mu \mathrm{m} \times 42 \mu \mathrm{m} \times 16 \mu \mathrm{m}$. The printing process is as follows. First, the structure information is sent to the printer controller in a CAD file. Next, the controller converts the structure information into a series of $16 \mu \mathrm{m}$ thick layered slices. The layer information is then sent to the printer. According to the received data, printer heads print a layered polymer structure. The jetted polymer is cured immediately by the ultraviolet lamps on the print heads. In a layer-by-layer fashion from bottom to top, a 3-D structure is finally produced. Eden 350 allows simultaneous printing of two types of materials conveniently, in this work, specifically, the 3-D-printed polymer material (VeroBlackPlus RGD875) and the support material (FullCure 705). The support material fills in any gap and prevents the polymer from drooping during printing. Once the entire structure is printed, the support material can be removed easily by high-pressure water spray. Fig. 13(a) shows the 3-D-printed polymer structure. The complete antenna prototype with the metallic cover is shown in Fig. 13(b).
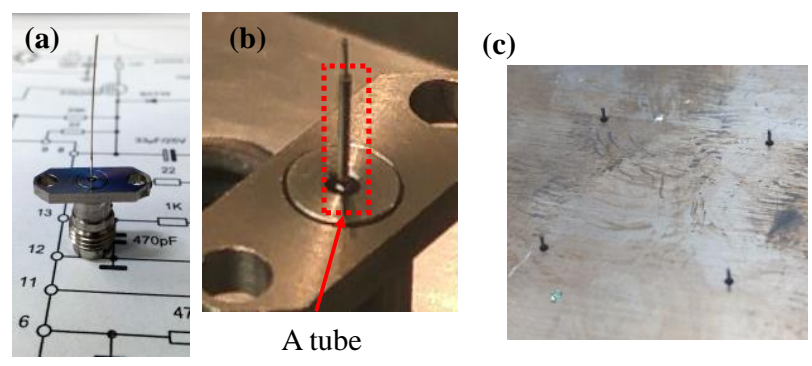

Fig. 12. (a) The V-connector with the pin soldered. (b) The pin is inserted into the tube, which acts as a positioner during connectors' attachment to the ground to make sure that the pins are at the center of the holes. (c) The fabricated four monopoles on the ground plane.
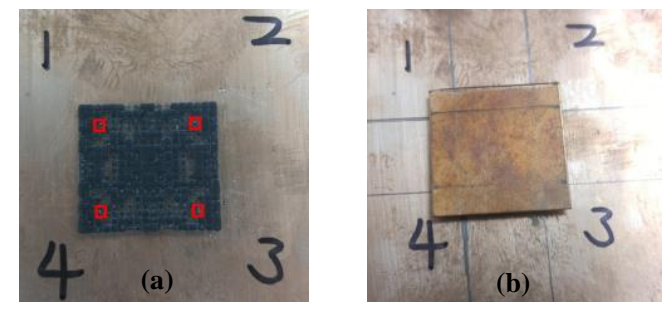

Fig. 13. (a) The four monopoles (indicated by the red squares) with the 3-D-printed polymer surrounding. (b) The final antenna structure with the metallic cover. The ground is $152.4 \times 152.4 \mathrm{~mm}^{2}$ and $0.4 \mathrm{~mm}$ thick. The dielectric is $18 \times 18 \times 1.25 \mathrm{~mm}^{3}$. The four monopoles are at vertices of a $12 \times 12$ $\mathrm{mm}^{2}$ square. The top metallic cover has a dimension of $20 \times 20 \times 0.4 \mathrm{~mm}^{3}$.

\section{B. Measurement Results}

The measured $S_{11}$ of the monopoles only case is illustrated in Fig. 14. It is observed that the measured resonance frequencies are lower than those of simulation. This is due to the extra length of the monopoles as a result of fabrication inaccuracies. It is discovered that the measurement results are closest to the simulation results of 1.16-mm-long monopoles, as shown in Fig. 15. $S_{12}, S_{34}, S_{14}, S_{32}, S_{31}$, and $S_{24}$ are then measured and compared with the 1.16-mm-long monopole simulation results, both with and without the dielectric loading and the metallic cover, as depicted in Fig. 16. In general, good agreement between the measurement and simulation results is observed. The discrepancies, especially the resonant frequency shift of $\mathrm{S}_{14}$ and $\mathrm{S}_{32}$ in Fig. 16(c) and (d), are likely due to the position inaccuracies of the loaded dielectric and top metallic cover with respect to the antennas. In the experiment, it is quite hard to control the relative position accurately. To understand this better, simulation results for the case that the loaded dielectric is offset by $(-0.3 \mathrm{~mm}, 0.3 \mathrm{~mm}, 0 \mathrm{~mm})$ and the metallic cover is offset by $(0.5 \mathrm{~mm}, 0.5 \mathrm{~mm}, 0 \mathrm{~mm})$ are shown in Fig. 17. It can be observed that $S_{14}$ and $S_{32}$ agree better with the measurement results.

Moreover, Fig. 16(e) and (f) show that transmission loss as small as $15 \mathrm{~dB}$ is achieved at $60 \mathrm{GHz}$ at a distance of $17 \mathrm{~mm}$ in experiment, in comparison with $22.2 \mathrm{~dB}$ calculated by the Friis transmission equation for $60 \mathrm{GHz}$ monopoles and the reported $25 \mathrm{~dB}$ at $60 \mathrm{GHz}$ at a distance of $15 \mathrm{~mm}$ in [29] using zig-zag antennas. The experiment results have substantiated previous 
analysis and demonstrated the improvement in the desired links and suppression in crosstalk from 55 - $61 \mathrm{GHz}$.

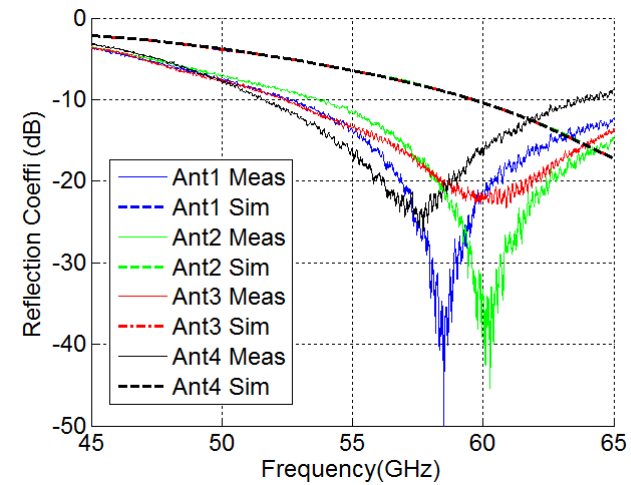

Fig. 14. The measured reflection coefficients of the monopoles only case in comparison with simulation results.

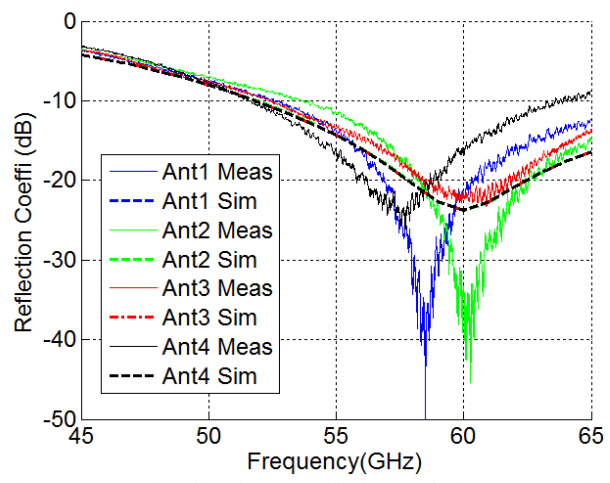

Fig. 15. The measured reflection coefficient of the monopoles only case in comparison with the simulation results of $1.16 \mathrm{~mm}$-long monopoles.

\section{ANALYSIS AND DISCUSSION}

Electromagnetic wave has slower propagating velocity in dielectrics than in air. Therefore, more time delay is expected for antennas surrounded by dielectrics compared with those in air. In this section, a quantitative comparison of the delay between the traditional RC line interconnects and the dielectric loaded antennas is performed.

The larger time delay in traditional IC interconnect is due to the increased resistance as a result of interconnect scaling. A study of the interconnect delay has been reported in [3]. Taking $1 \mathrm{~mm}$ path as an example, the RC delay is $\sim 80 \mathrm{ps}$ for the $500 \mathrm{~nm}$ process (heavily doped silicon); for the $35 \mathrm{~nm}$ process $(\mathrm{Cu}$, low $\mathrm{k})$, about $250 \mathrm{ps}$ delay is experienced. In comparison, the maximum delay for the wireless interconnect based on the proposed method is only $\tau=\frac{l \sqrt{\varepsilon_{r}}}{c}=5.5 \mathrm{ps}$ where $l=1 \mathrm{~mm}$ is the distance between antennas, $c$ is the speed of light, and $\varepsilon_{r}=$ 2.7 is the maximal possible dielectric constant of the dielectric loading. That is to say, for $1 \mathrm{~mm}$ distance, the wireless interconnect will be 50 times faster than the $\mathrm{RC}$ line interconnect for the $35 \mathrm{~nm}$ process. Moreover, the RC delay is proportional to the square of the distance [3]. In comparison, the wireless interconnect experiences delay proportional to the distance. Therefore, for larger distance, e.g., $17 \mathrm{~mm}$ communications, the wireless interconnect with loaded dielectric features about 850 times less delay compared to the $\mathrm{RC}$ line interconnect. In conclusion, the wireless interconnect with loaded dielectric is advantageous with significantly reduced time delay.
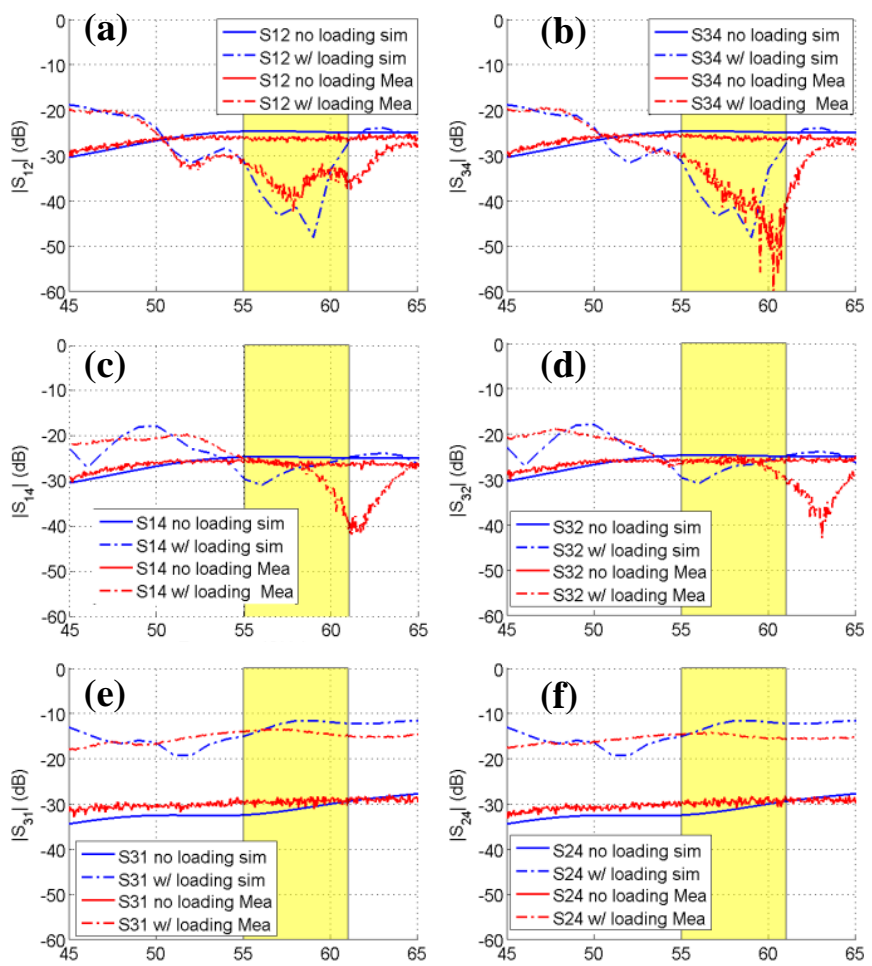

Fig. 16. The measured $S$-parameters of the fabricated monopoles in comparison with the simulation, with and without dielectric loading or metallic cover. (a) $S_{12}$; (b) $S_{34}$; (c) $S_{14}$; (d) $S_{32}$; (e) $S_{31}$; (f) $S_{24}$.
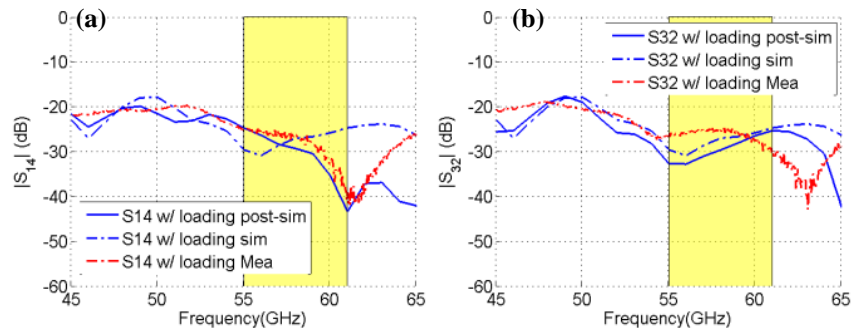

Fig. 17. The simulated $S$-parameters when the loaded dielectric is offset by $(-0.3 \mathrm{~mm}, 0.3 \mathrm{~mm}, 0 \mathrm{~mm})$ and the metallic cover is offset by $(0.5 \mathrm{~mm}, 0.5 \mathrm{~mm}$, $0 \mathrm{~mm}$ ). (a) $S_{14}$; (b) $S_{32}$.

Some interesting aspects of this work are worth further investigations. First, experimental validation of monopole antennas on chip are desired. One possible method is the direct ink writing of 3-D metal structure [30]. Second, this work may lend itself to reconfigurable wireless interconnect if a tunable dielectric material is available for loading and dynamic control.

\section{CONCLUSION}

Vertical quarter-wavelength monopoles surrounded by optimized dielectric distribution are proposed for future intra-chip wireless interconnects. As an example, the dielectric constant distribution surrounding four $160-\mathrm{GHz}$ monopoles is optimized by genetic algorithms to improve the desired wireless link and suppress crosstalk. The simulation shows that the desired link is improved by $8-15 \mathrm{~dB}$ and the crosstalk is reduced by $9-23 \mathrm{~dB}$ from $155 \mathrm{GHz}$ to $165 \mathrm{GHz}$ with our design. As an experimental proof-of-concept, a $60 \mathrm{GHz}$ prototype is designed, fabricated and measured. The 3-D printing technology is utilized to realize the designed $\varepsilon_{\mathrm{r}}$ distribution 
based on effective medium approximation, which features fast and convenient prototyping. The measurement results agree well with the simulation results, which demonstrate 10-18 dB improvement in the desired links and 10-30 dB reduction in crosstalk from 55 to $61 \mathrm{GHz}$. The transmission loss as small as $15 \mathrm{~dB}$ is achieved at $60 \mathrm{GHz}$ at a distance of $15 \mathrm{~mm}$ in the experiment, in comparison with $22.6 \mathrm{~dB}$ calculated by the Friis transmission equation for $60 \mathrm{GHz}$ monopoles and the reported $25 \mathrm{~dB}$ at $60 \mathrm{GHz}$ at a distance of $15 \mathrm{~mm}$ in [29] using zig-zag antennas. This work demonstrates that future wireless intra-chip communication could benefit from 3-D-engineered dielectric loading as part of antenna design methodology. It also unveils the promising potential of additive 3-D printing technology in wireless communication.

\section{REFERENCES}

[1] "Fab 42: Our Recent Announcement," Intel Newsroom. [Online]. Available:

https://newsroom.intel.com/editorials/fab-42-recent-announcement/. [Accessed: 26-Jun-2017].

[2] J. A. Davis et al., "Interconnect limits on gigascale integration (GSI) in the 21 st century," Proc. IEEE, vol. 89, no. 3, pp. 305-324, Mar. 2001

[3] K. C. Saraswat and F. Mohammadi, "Effect of Scaling of Interconnections on the Time Delay of VLSI Circuits," IEEE J. Solid-State Circuits, vol. 17, no. 2, pp. 275-280, Apr. 1982.

[4] M. T. Bohr and Y. A. El-Mansy, "Technology for advanced high-performance microprocessors," IEEE Trans. Electron Devices, vol. 45, no. 3, pp. 620-625, Mar. 1998.

[5] S. Deb, A. Ganguly, K. Chang, P. Pande, B. Beizer, and D. Heo, "Enhancing performance of network-on-chip architectures with millimeter-wave wireless interconnects," presented at the Application-specific Systems Architectures and Processors (ASAP), 201021 st IEEE International Conference on, 2010, pp. 73-80.

[6] M.-C. F. Chang, V. P. Roychowdhury, L. Zhang, H. Shin, and Y. Qian, "RF/wireless interconnect for inter- and intra-chip communications," Proc. IEEE, vol. 89, no. 4, pp. 456-466, 2001.

[7] L. P. Carloni, P. Pande, and Y. Xie, "Networks-on-chip in emerging interconnect paradigms: Advantages and challenges," presented at the 3rd ACM/IEEE International Symposium on Networks-on-Chip, 2009. NoCS 2009, 2009, pp. 93-102.

[8] S.-B. Lee et al., "A scalable micro wireless interconnect structure for CMPs," 2009, pp. 217-228.

[9] D. DiTomaso, A. Kodi, D. Matolak, S. Kaya, S. Laha, and W. Rayess, "Energy-efficient adaptive wireless NoCs architecture," in Networks on Chip (NoCS), 2013 Seventh IEEE/ACM International Symposium on, 2013, pp. 1-8.

[10] A. Ganguly, K. Chang, S. Deb, P. P. Pande, B. Belzer, and C. Teuscher, "Scalable Hybrid Wireless Network-on-Chip Architectures for Multicore Systems," IEEE Trans. Comput., vol. 60, no. 10, pp. 1485-1502, 2011.

[11] H. M. Cheema and A. Shamim, "The last barrier: on-chip antennas," IEEE Microw. Mag., vol. 14, no. 1, pp. 79-91, 2013.

[12] A. Babakhani, X. Guan, A. Komijani, A. Natarajan, and A. Hajimiri, "A 77-GHz Phased-Array Transceiver With On-Chip Antennas in Silicon: Receiver and Antennas," IEEE J. Solid-State Circuits, vol. 41, no. 12, pp. 2795-2806, 2006.

[13] A. Shamim, L. Roy, N. Fong, and N. G. Tarr, "24 GHz On-Chip Antennas and Balun on Bulk Si for Air Transmission," IEEE Trans. Antennas Propag., vol. 56, no. 2, pp. 303-311, 2008.

[14] S.-S. Hsu, K.-C. Wei, C.-Y. Hsu, and H. Ru-Chuang, "A 60-GHz Millimeter-Wave CPW-Fed Yagi Antenna Fabricated by Using 0.18CMOS Technology," IEEE Electron Device Lett., vol. 29, no. 6, pp. 625627, 2008.

[15] B. A. Floyd and C.-M. Hung, "Intra-chip wireless interconnect for clock distribution implemented with integrated antennas, receivers, and transmitters," Solid-State Circuits IEEE J. Of, vol. 37, no. 5, pp. 543-552, 2002.

[16] S. Pan and F. Capolino, "Design of a CMOS On-Chip Slot Antenna With Extremely Flat Cavity at $140 \mathrm{GHz}$," IEEE Antennas Wirel. Propag. Lett., vol. 10, pp. 827-830, 2011.
[17] M. R. Nezhad-Ahmadi, M. Fakharzadeh, B. Biglarbegian, and S. Safavi-Naeini, "High-Efficiency On-Chip Dielectric Resonator Antenna for mm-Wave Transceivers," IEEE Trans. Antennas Propag., vol. 58, no. 10, pp. 3388-3392, 2010.

[18] M. A. I. Sikder, A. K. Kodi, M. Kennedy, S. Kaya, and A. Louri, "OWN: Optical and Wireless Network-on-Chip for Kilo-core Architectures," in 2015 IEEE 23rd Annual Symposium on High-Performance Interconnects (HOTI), 2015, pp. 44-51.

[19] J. Wu, X. Yu, M. Liang, and H. Xin, "Antenna radiation pattern control through 3D printed inhomogeneous dielectrics," in Radio Science Meeting (Joint with AP-S Symposium), 2015 USNC-URSI, 2015, pp. 353-353.

[20] M. Liang, W.-R. Ng, K. Chang, K. Gbele, M. E. Gehm, and H. Xin, “A 3-D Luneburg Lens Antenna Fabricated by Polymer Jetting Rapid Prototyping," IEEE Trans. Antennas Propag., vol. 62, no. 4, pp. 17991807, Apr. 2014.

[21] A. M. Nicolson and G. F. Ross, "Measurement of the Intrinsic Properties of Materials by Time-Domain Techniques," IEEE Trans. Instrum. Meas., vol. 19 , no. 4, pp. 377-382, Nov. 1970.

[22] W. B. Weir, "Automatic measurement of complex dielectric constant and permeability at microwave frequencies," Proc. IEEE, vol. 62, no. 1, pp. 33-36, Jan. 1974.

[23] M. Liang and H. Xin, "Three-Dimensionally Printed/Additive Manufactured Antennas," in Handbook of Antenna Technologies, Z. N. Chen, Ed. Springer Singapore, 2015, pp. 1-30.

[24] Z. Wu, J. Kinast, M. E. Gehm, and H. Xin, "Rapid and inexpensive fabrication of terahertz electromagnetic bandgap structures," Opt. Express, vol. 16, no. 21, pp. 16442-16451, Oct. 2008.

[25] Z. Wu, M. Liang, W.-R. Ng, M. Gehm, and H. Xin, "Terahertz Horn Antenna Based on Hollow-Core Electromagnetic Crystal (EMXT) Structure," IEEE Trans. Antennas Propag., vol. 60, no. 12, pp. 55575563, Dec. 2012.

[26] Z. Wu, W.-R. Ng, M. E. Gehm, and H. Xin, "Terahertz electromagnetic crystal waveguide fabricated by polymer jetting rapid prototyping," Opt. Express, vol. 19, no. 5, pp. 3962-3972, Feb. 2011.

[27] W.-R. Ng, D. R. Golish, H. Xin, and M. E. Gehm, "Direct rapid-prototyping fabrication of computer-generated volume holograms in the millimeter-wave and terahertz regime," Opt. Express, vol. 22, no. 3, pp. 3349-3355, Feb. 2014.

[28] P. Nayeri et al., "3D Printed Dielectric Reflectarrays: Low-Cost High-Gain Antennas at Sub-Millimeter Waves," IEEE Trans. Antennas Propag., vol. 62, no. 4, pp. 2000-2008, Apr. 2014.

[29] X. Yu, S. P. Sah, B. Belzer, and D. Heo, "Performance evaluation and receiver front-end design for on-chip millimeter-wave wireless interconnect," in Green Computing Conference, 2010 International, 2010, pp. 555-560.

[30] M. A. Skylar-Scott, S. Gunasekaran, and J. A. Lewis, "Laser-assisted direct ink writing of planar and 3D metal architectures," Proc. Natl. Acad. Sci., vol. 113, no. 22, pp. 6137-6142, May 2016. 